\title{
COMPLEX STATIONARY CONVECTION WITH THIRD-KIND BOUNDARY CONDITIONS AT THE BOUNDARIES OF A FLUID LAYER
}

\author{
A. V. Gorshkov*, E. Y. Prosviryakov \\ Institute of Engineering Science, Ural Branch of the Russian Academy of Sciences, 34 Komsomolskaya st., \\ Ekaterinburg, Russian Federation \\ *Corresponding author. E-mail: alex55gor@mail.ru; address for correspondence: 34 Komsomolskaya st., Ekaterinburg,
Russian Federation. Tel.: +7 (343) 375-35-92; fax: 374-53-30
}

In this paper presents analytical solutions of the Oberbeck-Boussinesq system describing layered Benard-Marangoni convection flows in an incompressible viscous fluid. On the free surface a third-kind boundary condition is used, namely Newton's heat transfer law. conditions.

Keywords: Benard-Marangoni convection, analytical solution, third-kind boundary

DOI: $10.17804 / 2410-9908.2016 .2 .034-047$

\section{References}

1. Thomson J. On certain curious motions observable on the surfaces of wine and other alcoholic liquours. The London, Edinburgh, and Dublin Philosophical Magazine and Journal of Science, vol. X, fourth series. London, Taylor and Francis, Red Lion Court, Fleet Street, 1855, pp. 330-333.

2. Bénard H. Les Tourbillons cellulaires dans une nappe liquide propageant de la chaleur par convection, en régime permanent. Paris, Gauthier-Villars, 1901.

3. B'enard H. Etude exp'erimentale des courants de convection dans une nappe liquide. R'egime permanent: tourbillons cellulaires. J. Phys. Theor. Appl., 1900, vol. 9, no. 1, pp. 513-524.

4. Getling A.V. Formation of the spatial structures of Rayleigh-Benard convection. Uspekhi Fizicheskikh Nauk, 1991, vol. 161, no. 9, pp. 1-80. (In Russian).

5. Landa P.S. Avtokolebaniya v raspredelennykh sistemakh [Self-Excited Vibrations in Distribution Systems]. M., Knizhnyi Dom Publ., 2010, pp. 320. (In Russian).

6. Marangoni C. Sull espansione delle goccie di un liquido galleggiante sulla superficie di altro liquid. Pavia: Tipografia dei fratelli Fusi, 1865.

7. Birikh R.V. Thermocappillary convection on a horizontal fluid layer. J. Appl. Mech. Technol. Phys., 1966, vol. 7, iss. 3, pp. 43-44. DOI: 10.1007/BF00914697.7.

8. Ostroumov G.A. Svobodnaya konvektsiya $v$ usloviyakh vnutrenney zadachi [Free Convection under Interior Problem Conditions]. M.; L., Gostekhteoretizdat Publ., 1952, 256 p. (In Russian).

9. Gershuni G.Z., Zhukhovitsky E.M. Konvektivnaya ustoichivost neszhimaemoy zhidkosti [Convective Stability of Incompressible Fluid]. M., Nauka Publ., 1972. (In Russian).

10. Napolitano L.G. Plane Marangoni-Poiseuille flow of two immissible fluids. Acta Astronautica, 1980, vol. 7, iss. 4-5, pp. 461-478. DOI: 10.1016/0094-5765(80)90036-3.

11. Sidorov A.F. On one class of solutions to equations of gas dynamics and natural convection. In: Chislennye $i$ analiticheskie metody resheniya zadach sploshnoi sredy [Numerical and Analytical Methods of Solving Continuum Problems]. Sverdlovsk, UNTs AN SSSR Publ., 1981, pp. 101-117. (In Russian).

12. Sidorov A.F. Two classes of solution of the fluid and gas mechanics equatins and their connection to traveling wave theory. Journal of Applied Mechanics and Technical Physics, 1989, vol. 30, iss. 2, pp. 197-203. DOI: 10.1007/BF00852164. 
13. Kirdyashkin A.G. Teplovye gravitatsionnye techeniya $i$ teploobmen $v$ astenosfere [Heat and Gravitational Flows and Heat Exchange in the Asthenosphere]. Novosibirsk, Nauka, SO RAN Publ., 1989, 81 p. (In Russian).

14. Goncharova O., Kabov O. Gas flow and thermocapillary effects of fluid flow dynamics in a horizontal layer. Microgravity Sci. Technol., 2009, vol. 21, iss. 1 suppl., pp. 129-137. DOI: $10.1007 / \mathrm{s} 12217-009-9108-\mathrm{x}$.

15. Andreev V.K. Resheniya Birikha uravneniy konvektsii i nekotorye ego obobshcheniya [Birikh Solutions to Convection Equations and Some of its Extensions]. Krasnoyarsk, IBM SO RAN Publ., 2010, 68 p. (In Russian).

16. Ingel L.Kh., Kalashnik Maksim V. Nontrivial features in the hydrodynamics of seawater and other stratified solutions. Physics-Uspekhi, vol. 55, no. 4, pp. 356-381. DOI: $10.3367 /$ UFNe.0182.201204b.0379.

17. Aristov S.N. Shvarts K.G. Vikhrevye techeniya v tonkikh sloyakh zhidkosti [Vortex Flows in Thin Fluid Layers]. Kirov, VyatGU Publ., 2011, 207 p. (In Russian).

18. Aristov S.N., Prosviryakov E.Yu. On layered flows of plane free convection. Nelineynaya dinamika, 2013, vol. 9, no. 4, pp. 651-657. (In Russian).

19. Shvarts K.G. Plane-parallel advective flow in a horizontal layer of incompressible fluid with solid boundaries. Izvestiya RAN. MZhG, 2014, no. 4, pp. 26-30. (In Russian).

20. Yudovich V.I. On the challenges and prospects of advanced mathematical hydrodynamics. Uspekhi mekhaniki, 2002, vol. 1, no. 1, pp. 61-102. (In Russian).

21. Aristov S.N., Shvarts K.G. Vikhrevye techeniya advektivnoy prirody vo vrashchayushchemsya sloe zhidkosti [Vortex Flows of Advective Nature in a Rotating Fluid Layer]. Perm, Izd-vo PGU Publ., 2006, 154 p. (In Russian).

22. Aristov S.N., Shvarts K.G. Advective flow in a rotating liquid film. Journal of Applied Mechanics and Technical Physics, 2016, vol. 57, no. 1, pp. 188-194. DOI: $10.1134 / \mathrm{S} 0021894416010211$.

23. Andreev V.K., Bekezhanova V.B. Stability of non-isothermal fluids (Review). Journal of Applied Mechanics and Technical Physics, 2013, vol. 54, iss. 2, pp. 171-184. DOI: $10.1134 / \mathrm{S} 0021894413020016$.

24. Pukhnachev V.V. Non-stationary analogs of the Birikha solution. Izvestiya Altaiskogo gos. Universiteta, 2011, no. 1-2, pp. 62-69. (In Russian).

25. Nikitin N.V., Nikitin S.A., Polezhaev V.I. Convective instabilities in the hydrodynamic model of crystal growth by the Chokhralsky method. Uspekhi Mekhaniki, 2003, vol. 2, no. 4, pp. 63-105. (In Russian).

26. Aristov S.N., Zimin V.D. Advektivnye volny vo vrashchayushchemsya sharovom sloe [Adequate Waves in a Rotating Spherical Layer]. IMMS, UNTs, AN SSSR Publ., Sverdlovsk, 1986, 50 p. (In Russian).

27. Aristov S.N., Frik P.G. Dinamika krupnomasshtabnykh techeniy v tonkikh sloyakh zhidkosti [Dynamics of Large-Scale Flows in Thin Fluid Layers]. IMMS, UNTs, AN SSSR Publ., Sverdlovsk, 1987, 48 p. (In Russian).

28. Aristov S.N., Shvarts K.G. Convective heat exchange in a plane layer of incompressible fluid under localized heating. Izvestiya RAN. MZhG, 2013, no. 3, pp. 53-58. (In Russian).

29. Aristov S.N., Prosviryakov E.Yu. On one class of analytical solutions to stationary axisymmetric Benard-Marangoni convection of viscous incompressible fluid. Vestnik Sam. gos. Un-ta. Ser. fiz.-mat. nauki, 2013, no. 3 (32), pp. 110-118. (In Russian).

30. Aristov S.N., Knyazev D.V. Localized convective flows in a layer of nonuniformly heated fluid. Izvestiya RAN. MZhG, 2014, no. 5, pp. 5. (In Russian).

31. Aristov S.N., Frik P.G. Large-scale turbulency in a thin layer of nonisothermal rotating fluid. Izvestiya RAN SSSR. MZhG, 1988, no. 4, pp. 48-55. (In Russian). 
32. Landau L.D. Teoreticheskaya fizika. T. 6. Gidrodinamika [Theoretical Physics. Vol. 6. Hydrodynamics]. M., Nauka Publ., 2006, 736 p. (In Russian).

33. Lin C.C. Note on a class of exact solutions in magneto-hydrodynamics. Arch. Rational Mech. Anal., 1958, vol. 1, pp. 391-395.

34. Aristov S.N., Knyazev D.V., Polyanin A.D. Exact solutions to Navier-Stokes equations with the linear dependence of the velocity components on two spatial variables. Teoreticheskie osnovy khim. tekhnologii, 2009, vol. 43, no. 5, pp. 547-566. (In Russian).

35. Gantmakher F.R. Teoriya matrits [Theory of Matrices]. M., Nauka Publ., 1967, 576 p. (In Russian). 
Подана в журнал: 30.03 .2016

УДК 539

DOI: $10.17804 / 2410-9908.2016 .2 .034-047$

\title{
СТАЦИОНАРНАЯ СЛОЖНАЯ КОНВЕКЦИЯ ПРИ ГРАНИЧНЫХ УСЛОВИЯХ ІІІ РОДА НА ГРАНИЦАХ СЛОЯ ЖИДКОСТИ
}

\author{
А. В. Горшков*, Е. Ю. Просвиряков \\ Федеральное государственное бюджетное учреждение науки Институт машиноведения \\ Уральского отделения Российской академии наук, ул. Комсомольская, 34, Екатеринбург, Российская Федерация \\ *Ответственный автор. Электронная почта: alex55gor@mail.ru; адрес для переписки: ул. Комсомольская, 34, \\ Екатеринбург, Российская Федерация. Телефон: +7 (343) 375-35-92; факс: 374-53-30
}

В работе получены аналитические решения системы Обербека - Буссинеска, описывающие слоистые течения конвекции Бенара - Марангони в несжимаемой вязкой жидкости. На границах слоя жидкости используется граничное условие третьего рода - закон теплопередачи по Ньютону.

Ключевые слова: конвекция Бенара - Марангони, аналитическое решение, граничные условия III рода.

\section{1. Введение}

Изучение конвективных течений вязкой несжимаемой жидкости является одной из наиболее распространенных задач в самых разных теоретических и прикладных научных дисциплинах. В последние десятилетия интерес к исследованию решений, описывающих естественную конвекцию, непрерывно возрастает. Это связано с тем, что конвекция является первым примером самоорганизации динамических систем, наблюдаемых экспериментально [1]. Первая именная структура самоорганизации также относится к конвекции - ячейки Бенара, представляющие собой шестиугольные призмы с подъемом жидкости в центре [2-4].

Уже в первых гидродинамических опытах [2-4] Бенаром было высказано предположение о том, что важную роль в возникновении конвекции играет не только сила тяжести, а термокапиллярный эффект Марангони [5]. Отметим, что исследование эффекта Марангони вносит ясность не только в термокапиллярную конвекцию, но и в концентрационную конвекцию и биоконвекцию [6].

При моделировании указанных выше процессов движения жидкости очень важно иметь определенный запас точных решений, потому что полное интегрирование уравнений Навье - Стокса в приближении Бусснеска (система Обербека - Буссинеска) является до сих пор открытой проблемой. Первое точное решение, описывающее термокапиллярные движения вязкой несжимаемой жидкости, было предложено Бирихом [7]. Пионерским точным конвективным решением является течение Остроумова, обладающее вращательной симметрией [8]. Наиболее полный список точных конвективных движений можно изучить по работам [9-31] и библиографическим спискам к ним.

В работе предложено новое точное решение, описывающее стационарную термокапиллярную конвекцию при реализации на границах слоя жидкости теплообмена по закону Ньютона - Рихмана [9]. Исследование таких движений вязкой несжимаемой жидкости чрезвычайно важно для практических задач. Даже при проведении лабораторных экспериментальных исследований всегда трудно достичь теплоизолированной границы для замкнутых сосудов, а для термокапиллярных течений, наверное, невозможно. 
Характерной особенностью полученного нового точного решения является одномерность скоростей по координатам, а поля температуры и давления трехмерны по координатам. Выбранные условия соответствуют теоретическим экспериментальным исследованиям по гидродинамике [9-31]. При устойчивом движении неизотермической жидкости одномерность скоростей практически достигается в прямоугольном слое, что не наблюдается для температуры и давления даже в самых простых случаях [9].

\section{2. Постановка задачи}

Общая система, описывающая стационарную конвекцию в несжимаемой жидкости, в приближении Обербека - Буссинеска имеет вид [9, 32]:

$$
\begin{aligned}
& \mathrm{V}_{\mathrm{x}} \frac{\partial \mathrm{V}_{\mathrm{x}}}{\partial \mathrm{x}}+\mathrm{V}_{\mathrm{y}} \frac{\partial \mathrm{V}_{\mathrm{x}}}{\partial \mathrm{y}}+\mathrm{V}_{\mathrm{z}} \frac{\partial \mathrm{V}_{\mathrm{x}}}{\partial \mathrm{z}}=-\frac{\partial \mathrm{P}}{\partial \mathrm{x}}+v \Delta \mathrm{V}_{\mathrm{x}} ; \\
& \mathrm{V}_{\mathrm{x}} \frac{\partial \mathrm{V}_{\mathrm{y}}}{\partial \mathrm{x}}+\mathrm{V}_{\mathrm{y}} \frac{\partial \mathrm{V}_{\mathrm{y}}}{\partial \mathrm{y}}+\mathrm{V}_{\mathrm{z}} \frac{\partial \mathrm{V}_{\mathrm{y}}}{\partial \mathrm{z}}=-\frac{\partial \mathrm{P}}{\partial \mathrm{y}}+v \Delta \mathrm{V}_{\mathrm{y}} ; \\
& \mathrm{V}_{\mathrm{x}} \frac{\partial \mathrm{V}_{\mathrm{z}}}{\partial \mathrm{x}}+\mathrm{V}_{\mathrm{y}} \frac{\partial \mathrm{V}_{\mathrm{z}}}{\partial \mathrm{y}}+\mathrm{V}_{\mathrm{z}} \frac{\partial \mathrm{V}_{\mathrm{z}}}{\partial \mathrm{z}}=-\frac{\partial \mathrm{P}}{\partial \mathrm{z}}+v \Delta \mathrm{V}_{\mathrm{z}}+\mathrm{g} \beta \mathrm{T} ; \\
& \mathrm{V}_{\mathrm{x}} \frac{\partial \mathrm{T}}{\partial \mathrm{x}}+\mathrm{V}_{\mathrm{y}} \frac{\partial \mathrm{T}}{\partial \mathrm{y}}+\mathrm{V}_{\mathrm{z}} \frac{\partial \mathrm{T}}{\partial \mathrm{z}}=\chi \Delta \mathrm{T} ; \\
& \frac{\partial \mathrm{V}_{\mathrm{x}}}{\partial \mathrm{x}}+\frac{\partial \mathrm{V}_{\mathrm{y}}}{\partial \mathrm{y}}+\frac{\partial \mathrm{V}_{\mathrm{z}}}{\partial \mathrm{z}}=0 .
\end{aligned}
$$

В системе (1) используются стандартные обозначения: $\chi$ - коэффициент температуропроводности; $v$ - коэффициент кинематической вязкости жидкости; $\beta$ - коэффициент объемного расширения жидкости; $g$ - ускорение свободного падения; $\mathrm{V}_{\mathrm{x}}, \mathrm{V}_{\mathrm{y}}, \mathrm{V}_{\mathrm{z}}$ - компоненты вектора скорости жидкости.

Решения уравнений Обербека - Буссинеска вычислим для граничных условий, соответствующих конвективному движению Бенара - Марангони и теплообмену, определяемому условием Ньютона - Рихмана. В силу того, что рассматривается вязкая жидкость, то на нижней (недеформируемой) поверхности для скорости выполняются условия прилипания

$$
\mathrm{V}_{\mathrm{x}}(\mathrm{x}, \mathrm{y}, 0)=0 ; \quad \mathrm{V}_{\mathrm{y}}(\mathrm{x}, \mathrm{y}, 0)=0 ; \quad \mathrm{V}_{\mathrm{z}}(\mathrm{x}, \mathrm{y}, 0)=0 .
$$

На свободной поверхности, определяемой уравнением $z=h$, заданы условия термокапиллярной конвекции [9]:

$$
\left.\eta \frac{d U}{d n}\right|_{z=h}=\sigma T(x, y, h),
$$

где $\sigma<0$ - температурный коэффициент поверхностного натяжения; $\eta$ - динамическая вязкость жидкости. Граничные условия (3) записаны в предположении о неформируемости свободной поверхности при движении жидкости и линейной зависимости поверхностного натяжения от температуры [6, 7]: $\alpha(T)=\alpha_{0}+\sigma T$. 
На верхней границе $z=h$ и на нижней поверхности $z=0$ заданы условия теплопередачи по закону Ньютона - Рихмана [7, 9, 29]:

$$
\begin{aligned}
& \left.\lambda \frac{\partial \mathrm{T}}{\partial \mathrm{n}}\right|_{\mathrm{z}=\mathrm{h}}=\alpha_{1}\left(\mathrm{~T}_{\mathrm{Cl}}(\mathrm{x}, \mathrm{y})-\mathrm{T}(\mathrm{x}, \mathrm{y}, \mathrm{h})\right) \\
& \left.\lambda \frac{\partial \mathrm{T}}{\partial \mathrm{n}}\right|_{\mathrm{z}=0}=\alpha_{2}\left(\mathrm{~T}_{\mathrm{C} 2}(\mathrm{x}, \mathrm{y})-\mathrm{T}(\mathrm{x}, \mathrm{y}, 0)\right),
\end{aligned}
$$

где $T_{C 1}(x, y)=A_{1} x+B_{1} y$ - температура внешней среды; $T_{C 2}(x, y)=A_{2} x+B_{2} y$ - температура нижней поверхности; $A_{1}, B_{1}, A_{2}$ и $B_{2}$ - компоненты градиента температуры для свободной и недеформируемой границ соответственно; $\alpha_{1}, \alpha_{2}$ - коэффициенты теплопередачи.

Нормированное давление к плотности жидкости на внешней, свободной, поверхности имеет вид:

$$
P(x, y, h)=S=\frac{s}{\rho_{0}},
$$

где $s$ - атмосферное давление; $\rho_{0}$ - плотность жидкости.

\section{3. Результаты и обсуждение}

\section{1 Анализ разрешимости системы Обербека - Буссинеска для слоистой конвекции}

Далее рассмотрим слоистую конвекцию. Для реализации слоистых течений должно выполняться условие $V_{z}=0[13-15,18,19]$. Отметим, что в отличие от пионерской работы [7] здесь рассматривается не однонаправленный поток. Ниже будет показано, что при определенных условиях рассматриваемую краевую задачу нельзя свести к одномерной. При тождественном нулевом значении компоненты скорости $V_{z}$ система уравнений (1) становится переопределенной. Таким образом, для описания сложной конвекции структура класс решения должна быть такой, чтобы появилось одно «лишнее» уравнение, гарантирующее разрешимость задачи. Стационарное решение ищем в виде $[11,12,18,19,33,34]$ :

$$
\begin{aligned}
& V_{x}=U(z), V_{y}=V(z), \\
& T(x, y, z)=T_{0}(z)+x T_{1}(z)+y T_{2}(z), \\
& P(x, y, z)=P_{0}(z)+x P_{1}(z)+y P_{2}(z) .
\end{aligned}
$$

Отметим, что плоскопараллельное движение переопределенной системы Обербека Буссинеска в рамках класса (5) впервые было приведено в статье [18], где описаны аналитические выражения для различных типов граничных условий, которые используются при математическом моделировании адвективных потоков. 
Подставив выражения (5) для гидродинамических полей в уравнения (1), получим следующую систему обыкновенных дифференциальных уравнений, определяющую вид неизвестных функций (5), которые зависят от вертикальной координаты $z$ :

$$
\begin{gathered}
\frac{\mathrm{d}^{2} \mathrm{~T}_{1}}{\mathrm{dz}^{2}}=0, \quad \frac{\mathrm{d}^{2} \mathrm{~T}_{2}}{\mathrm{dz}^{2}}=0 ; \\
\frac{\mathrm{dP}_{1}}{\mathrm{dz}}=\mathrm{g} \beta \mathrm{T}_{1}, \quad \frac{\mathrm{dP}_{2}}{\mathrm{dz}}=\mathrm{g} \beta \mathrm{T}_{2} ; \\
\mathrm{v} \frac{\mathrm{d}^{2} \mathrm{U}}{\mathrm{dz}^{2}}=\mathrm{P}_{1}, \quad \mathrm{v} \frac{\mathrm{d}^{2} \mathrm{~V}}{\mathrm{dz}^{2}}=\mathrm{P}_{2} ; \\
\frac{\mathrm{d}^{2} \mathrm{~T}_{0}}{\mathrm{dz}^{2}}=\frac{1}{\chi}\left(\mathrm{UT}_{1}+\mathrm{VT}_{2}\right), \quad \frac{\mathrm{dP}_{0}}{\mathrm{dz}}=\mathrm{g} \beta \mathrm{T}_{0} .
\end{gathered}
$$

Уравнения в системе (6) выписаны в том порядке, в котором будет осуществляться интегрирование. Краевые условия (2) - (4) в силу структуры выражений (5) записываются следующим образом:

$$
\begin{gathered}
\mathrm{U}(0)=0 ; \quad \mathrm{V}(0)=0 ; \\
\left.\eta \frac{d U}{d z}\right|_{z=h}=\sigma T_{1}(h) ;\left.\eta \frac{d V}{d z}\right|_{z=h}=\sigma T_{2}(h) \\
\mathrm{P}_{1}(\mathrm{~h})=0 ; \quad \mathrm{P}_{2}(\mathrm{~h})=0 ; \quad \mathrm{P}_{0}(\mathrm{~h})=\mathrm{S} / \rho_{0} ; \\
\frac{\left.\partial T_{1}\right|_{z=0}=-\vartheta_{1}\left(A_{1}-T_{1}(0)\right) ;\left.\frac{\partial T_{2}}{\partial z}\right|_{z=0}=\vartheta_{2}\left(B_{1}-T_{2}(0)\right) ;}{\left.\frac{\partial T_{1}}{\partial z}\right|_{z=h}=\vartheta_{2}\left(A_{2}-T_{1}(h)\right) ;\left.\frac{\partial T_{2}}{\partial z}\right|_{z=h}=\vartheta_{2}\left(B 2-T_{2}(h)\right) ; T_{0}(x, y, h)=0 ; T_{0}(x, y, 0)=0,}
\end{gathered}
$$

где $\vartheta_{1}=\alpha_{1} / \lambda, \vartheta_{2}=\alpha_{2} / \lambda$.

Очевидно, что уравнение неразрывности, входящее в систему (1), при данном выборе представления решения выполняется тождественно.

\section{2 Вычисление точного речения краевой задачи (6)-(8)}

Решение для первых двух уравнений (6), определяющих функции $T_{1}$ и $T_{2}$, записывается в виде линейных функций:

$$
T_{1}=a_{1} z+b_{1}, T_{2}=a_{2} z+b_{2} .
$$

В результате вычислений получим коэффициенты решения, удовлетворяющие краевым условиям (8):

$$
\begin{aligned}
& \mathrm{a}_{1}=\frac{\left(\mathrm{A}_{2}-\mathrm{A}_{1}\right) \vartheta_{1} \vartheta_{2}}{\vartheta_{1}+\vartheta_{2}+\mathrm{h} \vartheta_{1} \vartheta_{2}} ; \quad \mathrm{b}_{1}=\frac{\mathrm{A}_{1} \vartheta_{1}+\mathrm{A}_{2} \vartheta_{2}+\mathrm{A}_{1} \mathrm{~h} \vartheta_{1} \vartheta_{2}}{\vartheta_{1}+\vartheta_{2}+\mathrm{h} \vartheta_{1} \vartheta_{2}} ; \\
& \mathrm{a}_{2}=\frac{\left(\mathrm{B}_{2}-\mathrm{B}_{1}\right) \vartheta_{1} \vartheta_{2}}{\vartheta_{1}+\vartheta_{2}+\mathrm{h} \vartheta_{1} \vartheta_{2}} ; \quad \mathrm{b}_{2}=\frac{\mathrm{B}_{1} \vartheta_{1}+\mathrm{B}_{2} \vartheta_{2}+\mathrm{B}_{1} \mathrm{~h} \vartheta_{1} \vartheta_{2}}{\vartheta_{1}+\vartheta_{2}+\mathrm{h} \vartheta_{1} \vartheta_{2}} .
\end{aligned}
$$


Таким образом, компоненты градиента температуры распределены по следующему закону:

$$
T_{1}=\frac{\vartheta_{1} \vartheta_{2}\left(A_{2}-A_{1}\right)}{\vartheta_{1}+\vartheta_{2}+h \vartheta_{1} \vartheta_{2}} z+\frac{A_{1} \vartheta_{1}+A_{2} \vartheta_{2}+h A_{1} \vartheta_{1} \vartheta_{2}}{\vartheta_{1}+\vartheta_{2}+h \vartheta_{1} \vartheta_{2}} ; T_{2}=\frac{\vartheta_{1} \vartheta_{2}\left(B_{2}-B_{1}\right)}{\vartheta_{1}+\vartheta_{2}+h \vartheta_{1} \vartheta_{2}} z+\frac{B_{1} \vartheta_{1}+B_{2} \vartheta_{2}+h B_{1} \vartheta_{1} \vartheta_{2}}{\vartheta_{1}+\vartheta_{2}+h \vartheta_{1} \vartheta_{2}}
$$

Компоненты градиента приведенного давления имеют параболический профиль:

$$
\begin{aligned}
& P_{1}=\frac{\vartheta_{1} \vartheta_{2}\left(A_{2}-A_{1}\right)}{\vartheta_{1}+\vartheta_{2}+h \vartheta_{1} \vartheta_{2}} \frac{\left(z^{2}-h^{2}\right)}{2}+\frac{A_{1} \vartheta_{1}+A_{2} \vartheta_{2}+h A_{1} \vartheta_{1} \vartheta_{2}}{\vartheta_{1}+\vartheta_{2}+h \vartheta_{1} \vartheta_{2}}(z-h) \\
& P_{2}=\frac{\vartheta_{1} \vartheta_{2}\left(B_{2}-B_{1}\right)}{\vartheta_{1}+\vartheta_{2}+h \vartheta_{1} \vartheta_{2}} \frac{\left(z^{2}-h^{2}\right)}{2}+\frac{B_{1} \vartheta_{1}+B_{2} \vartheta_{2}+h B_{1} \vartheta_{1} \vartheta_{2}}{\vartheta_{1}+\vartheta_{2}+h \vartheta_{1} \vartheta_{2}}(z-h) .
\end{aligned}
$$

Скорость при конвективном движении жидкости определяется многочленами, в общем случае имеющими четвертую степень:

$$
\begin{aligned}
& \mathrm{U}(\mathrm{z})=\frac{\left(\mathrm{A}_{2}-\mathrm{A}_{1}\right) \vartheta_{1} \vartheta_{2} \mathrm{z}^{4} \mathrm{~g} \beta}{24\left(\vartheta_{1}+\vartheta_{2}+\mathrm{h} \vartheta_{1} \vartheta_{2}\right) \mathrm{v}}+\frac{\mathrm{z}^{3} \mathrm{~g} \beta\left(\mathrm{A}_{1} \vartheta_{1}+\mathrm{A}_{2} \vartheta_{2}+\mathrm{A}_{1} \mathrm{~h} \vartheta_{1} \vartheta_{2}\right)}{6\left(\vartheta_{1}+\vartheta_{2}+\mathrm{h} \vartheta_{1} \vartheta_{2}\right) \mathrm{v}}- \\
& -\mathrm{z}^{2} \mathrm{hg} \beta \frac{\left(\mathrm{A}_{2}-\mathrm{A}_{1}\right) \mathrm{h} \vartheta_{1} \vartheta_{2}+2\left(\mathrm{~A}_{1} \vartheta_{1}+\mathrm{A}_{2} \vartheta_{2}+\mathrm{A}_{1} \mathrm{~h} \vartheta_{1} \vartheta_{2}\right)}{4\left(\vartheta_{1}+\vartheta_{2}+\mathrm{h} \vartheta_{1} \vartheta_{2}\right) \mathrm{v}}+ \\
& +\mathrm{z}\left[\mathrm{h}^{2} \mathrm{~g} \beta \frac{2\left(\mathrm{~A}_{2}-\mathrm{A}_{1}\right) \mathrm{h} \vartheta_{1} \vartheta_{2}+3\left(\mathrm{~A}_{1} \vartheta_{1}+\mathrm{A}_{2} \vartheta_{2}+\mathrm{A}_{1} \mathrm{~h} \vartheta_{1} \vartheta_{2}\right)}{6\left(\vartheta_{1}+\vartheta_{2}+\mathrm{h} \vartheta_{1} \vartheta_{2}\right) \mathrm{v}}-\right. \\
& \left.-\frac{\eta}{\sigma} \frac{\left(\left(\mathrm{A}_{2}-\mathrm{A}_{1}\right) \mathrm{h} \vartheta_{1} \vartheta_{2}+\left(\mathrm{A}_{1} \vartheta_{1}+\mathrm{A}_{2} \vartheta_{2}+\mathrm{A}_{1} \mathrm{~h} \vartheta_{1} \vartheta_{2}\right)\right)}{\left(\vartheta_{1}+\vartheta_{2}+\mathrm{h} \vartheta_{1} \vartheta_{2}\right)}\right] \\
& \mathrm{V}(\mathrm{z})=\frac{\left(\mathrm{B}_{2}-\mathrm{B}_{1}\right) \vartheta_{1} \vartheta_{2} \mathrm{z}^{4} \mathrm{~g} \beta}{24\left(\vartheta_{1}+\vartheta_{2}+\mathrm{h} \vartheta_{1} \vartheta_{2}\right) \mathrm{v}}+\frac{\mathrm{z}^{3} \mathrm{~g} \beta\left(\mathrm{B}_{1} \vartheta_{1}+\mathrm{B}_{2} \vartheta_{2}+\mathrm{B}_{1} \mathrm{~h} \vartheta_{1} \vartheta_{2}\right)}{6\left(\vartheta_{1}+\vartheta_{2}+\mathrm{h} \vartheta_{1} \vartheta_{2}\right) \mathrm{v}}- \\
& -\mathrm{g} \beta \mathrm{z}^{2} \frac{\left(\mathrm{B}_{2}-\mathrm{B}_{1}\right) \mathrm{h}^{2} \vartheta_{1} \vartheta_{2}+2 \mathrm{~h}\left(\mathrm{~B}_{1} \vartheta_{1}+\mathrm{B}_{2} \vartheta_{2}+\mathrm{B}_{1} \mathrm{~h} \vartheta_{1} \vartheta_{2}\right)}{4\left(\vartheta_{1}+\vartheta_{2}+\mathrm{h} \vartheta_{1} \vartheta_{2}\right) \mathrm{v}}+ \\
& +\mathrm{z}\left[\mathrm{h}^{2} \mathrm{~g} \beta \frac{2\left(\mathrm{~B}_{2}-\mathrm{B}_{1}\right) \mathrm{h} \vartheta_{1} \vartheta_{2}+3\left(\mathrm{~B}_{1} \vartheta_{1}+\mathrm{B}_{2} \vartheta_{2}+\mathrm{B}_{1} \mathrm{~h} \vartheta_{1} \vartheta_{2}\right)}{6\left(\vartheta_{1}+\vartheta_{2}+\mathrm{h} \vartheta_{1} \vartheta_{2}\right) \mathrm{v}}-\right. \\
& \left.-\frac{\eta}{\sigma} \frac{\left(\mathrm{B}_{2}-\mathrm{B}_{1}\right) \mathrm{h} \vartheta_{1} \vartheta_{2}+\mathrm{B}_{1} \vartheta_{1}+\mathrm{B}_{2} \vartheta_{2}+\mathrm{B}_{2} \mathrm{~h} \vartheta_{1} \vartheta_{2}}{\vartheta_{1}+\vartheta_{2}+\mathrm{h} \vartheta_{1} \vartheta_{2}}\right] .
\end{aligned}
$$


Температура $T_{0}$ имеет вид:

$$
\begin{aligned}
& \mathrm{T}_{0}=\chi\left\{\frac{\left(\mathrm{a}_{1}^{2}+\mathrm{a}_{2}^{2}\right) \mathrm{g} \beta}{1008 v} \mathrm{z}^{7}+\frac{\left(\mathrm{a}_{1} \mathrm{~b}_{1}+\mathrm{a}_{2} \mathrm{~b}_{2}\right) \mathrm{g} \beta}{144 v} \mathrm{z}^{6}+\right. \\
& +\mathrm{z}^{5}\left(\frac{\mathrm{g} \beta}{v} \frac{\mathrm{b}_{l}^{2}+\mathrm{b}_{2}^{2}}{120}-\mathrm{h} \frac{\mathrm{g} \beta}{v} \frac{\mathrm{a}_{l} \mathrm{~b}_{1}+\mathrm{a}_{2} \mathrm{~b}_{2}}{40}-\mathrm{h}^{2} \frac{\mathrm{g} \beta}{v} \frac{\mathrm{a}_{1}^{2}+\mathrm{a}_{2}^{2}}{80}\right)+ \\
& +\mathrm{z}^{4}\left[\mathrm{~h}^{3} \frac{\mathrm{g} \beta}{v} \frac{\mathrm{a}_{1}^{2}+\mathrm{a}_{2}^{2}}{36}+\mathrm{h}^{2} \frac{\mathrm{g} \beta}{v} \frac{\mathrm{a}_{1} \mathrm{~b}_{1}+\mathrm{a}_{2} \mathrm{~b}_{2}}{48}-\mathrm{h}\left(\frac{\eta}{\sigma} \frac{\mathrm{a}_{l}^{2}+\mathrm{a}_{2}^{2}}{12}+\frac{\mathrm{g} \beta}{v} \frac{\mathrm{b}_{1}^{2}+\mathrm{b}_{2}^{2}}{24}\right)-\frac{\eta}{\sigma} \frac{\mathrm{a}_{1} \mathrm{~b}_{1}+\mathrm{a}_{2} \mathrm{~b}_{2}}{12}\right]+ \\
& +\mathrm{z}^{3}\left[\frac{\mathrm{h}^{3} \beta \mathrm{g}}{v} \frac{\mathrm{a}_{l} \mathrm{~b}_{1}+\mathrm{a}_{2} \mathrm{~b}_{2}}{18}+\frac{\mathrm{h}^{2} \beta \mathrm{g}}{v} \frac{\mathrm{b}_{1}^{2}+\mathrm{b}_{2}^{2}}{12}-\mathrm{h} \frac{\eta}{\sigma} \frac{\mathrm{a}_{l} \mathrm{~b}_{1}+\mathrm{a}_{2} \mathrm{~b}_{2}}{6}-\frac{\eta}{\sigma} \frac{\mathrm{b}_{l}^{2}+\mathrm{b}_{2}^{2}}{6}\right]+ \\
& +\mathrm{z}\left[-41 \frac{\mathrm{h}^{6} \beta \mathrm{g}}{v} \frac{\mathrm{a}_{1}^{2}+\mathrm{a}_{2}^{2}}{2520}-\frac{\mathrm{h}^{5} \beta \mathrm{g}}{v} \frac{\mathrm{a}_{1} \mathrm{~b}_{1}+\mathrm{a}_{2} \mathrm{~b}_{2}}{120}-\frac{\mathrm{h}^{4} \beta \mathrm{g}}{v} \frac{\mathrm{b}_{1}^{2}+\mathrm{b}_{2}^{2}}{6}+\right. \\
& \left.+\frac{\sigma}{\eta}\left(\frac{\mathrm{h}^{2}}{6}\left(\mathrm{~b}_{1}^{2}+\mathrm{b}_{2}^{2}\right)+\frac{\mathrm{h}^{3}}{4}\left(\mathrm{a}_{1} \mathrm{~b}_{1}+\mathrm{a}_{2} \mathrm{~b}_{2}\right)+\frac{\mathrm{h}^{4}}{12}\left(\mathrm{a}_{1}^{2}+\mathrm{a}_{2}^{2}\right)\right)\right] \text {. }
\end{aligned}
$$

\section{3 Альтернативный подход к решению краевой задачи (6)-(8)}

Представим краевые условия на границах слоя жидкости как вектора градиента $\vec{T}_{g 1}$ и $\vec{T}_{g 2}$ температуры. В этом случае справедливы выражения:

$$
\begin{aligned}
& A_{1}=T_{g 1} \cos \psi, A_{2}=T_{g 2} \cos (\varphi+\psi) ; \\
& B_{1}=T_{g 1} \sin \psi, B_{2}=T_{g 2} \sin (\varphi+\psi) .
\end{aligned}
$$

Здесь $\varphi$ - угол между векторами $\vec{T}_{g 1}$ и $\vec{T}_{g 2} ; \psi-$ угол между вектором $\vec{T}_{g 1}$ и осью $o x$. В силу того, что рассматриваемая среда изотропна, решение зависит только от угла $\varphi$.

В этом случае коэффициенты $a_{1}, a_{2}, b_{1}, b_{2}$ примут вид:

$$
\begin{array}{ll}
a_{1}=\frac{\vartheta_{1} \vartheta_{2}\left(T_{g 2} \cos (\phi+\psi)-T_{g 1} \cos \psi\right)}{\vartheta_{1}+\vartheta_{2}+h \vartheta_{1} \vartheta_{2}} ; \quad b_{1}=\frac{T_{g 2} \vartheta_{2} \cos (\phi+\psi)+T_{g 1} \vartheta_{1}\left(1+h \vartheta_{2}\right) \cos \psi}{\vartheta_{1}+\vartheta_{2}+h \vartheta_{1} \vartheta_{2}}, \\
a_{2}=\frac{\vartheta_{1} \vartheta_{2}\left(T_{g 2} \sin (\phi+\psi)-T_{g 1} \sin \psi\right)}{\vartheta_{1}+\vartheta_{2}+h \vartheta_{1} \vartheta_{2}} ; \quad b_{2}=\frac{T_{g 2} \vartheta_{2} \sin (\phi+\psi)+T_{g 1} \vartheta_{1}\left(1+h \vartheta_{2}\right) \sin \psi}{\vartheta_{1}+\vartheta_{2}+h \vartheta_{1} \vartheta_{2}} .
\end{array}
$$

Здесь $T_{g 1}$ и $T_{g 2}$ соответственно модули векторов $\vec{T}_{g 1}$ и $\vec{T}_{g 2}$.

При повороте векторов $\vec{T}_{g 1}$ и $\vec{T}_{g 2}$ на угол $\psi$ соответственно повернется и вектор скорости $(U(z), V(z))$. 
Вычислим касательные напряжения на твердой поверхности:

$$
\begin{aligned}
& \tau_{z x}=\eta \frac{A_{2} \vartheta_{2}\left(6 \eta v\left(1+h \vartheta_{1}\right)+g h^{2} \beta\left(3+2 h \vartheta_{1}\right) \sigma\right)+A_{1} \vartheta_{1}\left(6 \eta v+g h^{2} \beta \sigma\left(3+h \vartheta_{2}\right)\right)}{6 v \sigma\left(\vartheta_{1}+\vartheta_{2}+h \vartheta_{1} \vartheta_{2}\right)} ; \\
& \tau_{z x}=\eta \frac{B_{2} \vartheta_{2}\left(6 \eta v\left(1+h \vartheta_{1}\right)+g h^{2} \beta\left(3+2 h \vartheta_{1}\right) \sigma\right)+B_{1} \vartheta_{1}\left(6 \eta v+g h^{2} \beta \sigma\left(3+h \vartheta_{2}\right)\right)}{6 v \sigma\left(\vartheta_{1}+\vartheta_{2}+h \vartheta_{1} \vartheta_{2}\right)} .
\end{aligned}
$$

Если векторы градиента температуры на границах слоя коллинеарны $\left(A_{2}, B_{2}\right)=\gamma\left(A_{1}, B_{1}\right)$, то существует толщина слоя жидкости, при которой касательные напряжения на твердой поверхности одновременно обращаются в нуль.

Если учесть связь векторов градиентов, уравнения $\tau_{z x}=0$ и $\tau_{z y}=0$, очевидно, совпадут, а следовательно, будут иметь одинаковые корни. Так как числители выражений $\tau_{z x}$ и $\tau_{z y}$ представляют собой кубичные многочлены относительно $h$, то один действительный корень всегда будет существовать. Для проверки условий его положительного значения можно использовать модификацию локализационных критериев проблемы Рауса - Гурвица [35].

При идеальном тепловом контакте на обеих границах условия $\tau_{z x}=0$ и $\tau_{z y}=0$ вырождаются к квадратным уравнениям с равным корнем:

$$
h_{2}=\sqrt{\frac{-6 \gamma \eta \nu}{\beta g \sigma(1+2 \gamma)}} .
$$

Вычисленный корень принимает положительные значения при

$$
\frac{-\gamma}{\sigma(1+2 \gamma)}>0
$$

Напомним, что справедливо неравенство $\sigma<0$. Таким образом, одновременное обращение в нуль касательных напряжений возможно, если

$$
\gamma \in\left(-\infty ;-\frac{1}{2}\right) \cup(0 ;+\infty) .
$$

Векторы градиента температуры должны быть всегда соноправлены или антинаправлены, но с определенным коэффициентом пропорциональности. Общего решения уравнений $\tau_{z x}=0$ и $\tau_{z y}=0$ при $\gamma \in\left(-\frac{1}{2} ; 0\right)$ не имеют. 
В качестве примера проведены расчеты скоростей $U, V$ и температуры $T_{0}$ для воды. Физические параметры взяты следующими:

$$
\begin{aligned}
& \beta=0,000210 \quad 1 / \mathrm{K} \\
& \alpha_{1}=350 \quad \text { вт } /\left(\mathrm{M}^{2} \mathrm{~K}\right) \text { коэффициент взят для пары сталь-вода } \\
& \alpha_{2}=5,6 \quad \text { вт } /\left(\mathrm{M}^{2} \mathrm{~K}\right) \text { коэффициент взят для пары вода-воздух } \\
& \vartheta_{1}=\alpha_{1} \mathrm{~h} / \lambda=583,333 ; \vartheta_{2}=\alpha_{2} \mathrm{~h} / \lambda=9,33333 \\
& \lambda=0,6 \mathrm{BT} /(\mathrm{M} \mathrm{K}) ; \chi=0,14310^{-6} \mathrm{~m}^{2} / \mathrm{ce \kappa} \\
& \eta=0,001 \text { Па сек; } v=0,000001 \mathrm{~m}^{2} / \text { сек } \\
& \mathrm{g}=9,81 \mathrm{~m} / \mathrm{ce \kappa}^{2} \\
& \sigma=-0,0727 \mathrm{n} / \mathrm{M}^{2} .
\end{aligned}
$$

При значении $\mathrm{h}=1$ численно определено значение параметра $\gamma=-0,659$, при котором касательные напряжения на твердой поверхности обращаются в ноль.

Изменение скорости $V$ по толщине в зависимости от параметра $B_{1}$ при значении параметра $\gamma=-0,659$ приведено на рис. 1 .

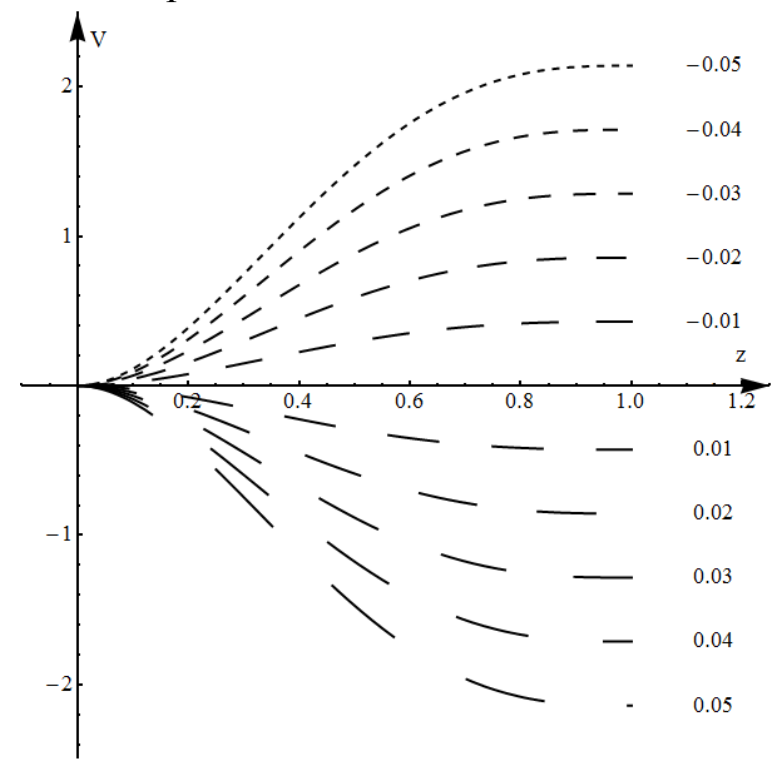

Рис. 1. Изменение скорости потока с глубиной при различных значениях параметра $B_{1}$

Из рисунка видно, что график скорости при $z=0$ касается оси $O z$, т.е. в точке $z=0$ касательные напряжения $\tau_{z x}$ и $\tau_{z y}$ обращаются в ноль. Графики скорости $U$ аналогичны приведенным для скорости $V$.

На рис. 2 представлено изменение скорости $U$ по толщине слоя в зависимости от коэффициента $\gamma$ при значении параметра $\mathrm{A}_{1}=0,3$. 


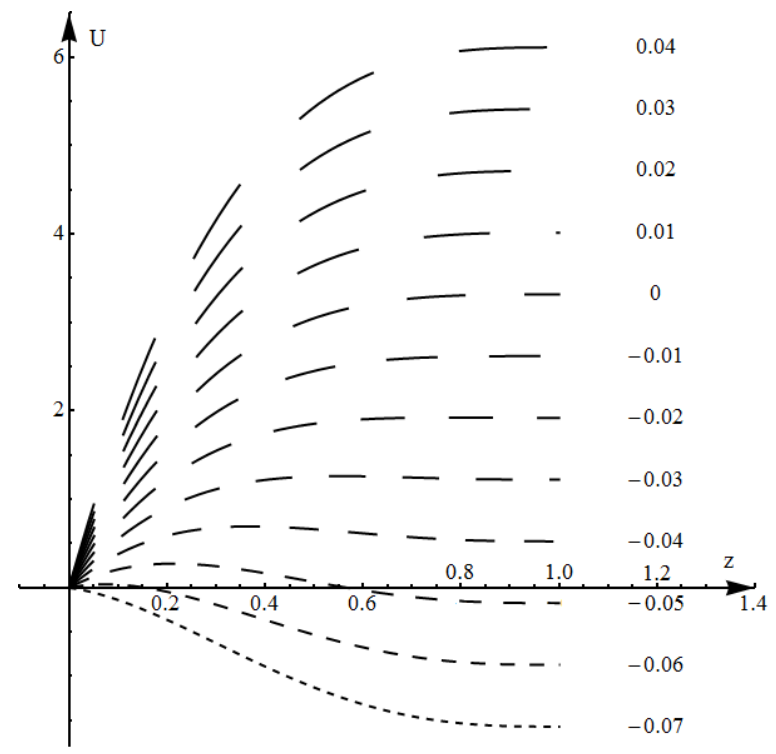

Рис. 2. Изменение скорости потока с глубиной при различных значениях параметра $\gamma$

При значении $\gamma=-0,05$ существует противоток. При значении $\gamma>-0,05$ противоток пропадает, при $\gamma<-0,05$ течение меняет направление.

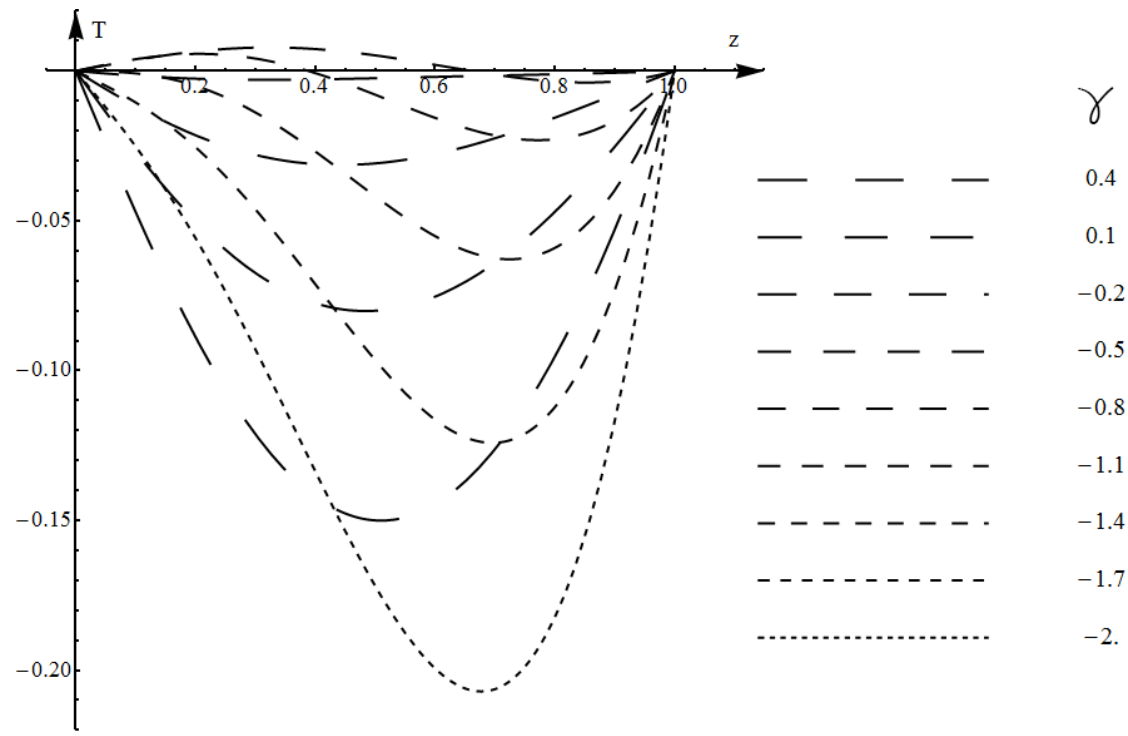

Рис. 3. Изменение температуры по глубине при различных значениях параметра $\gamma$

На рис. 3 представлено изменение температуры по толщине в зависимости от параметра $\gamma$, при $\mathrm{A}_{1}=-1 ; \mathrm{B}_{1}=0,1$. При больших и малых значениях $\gamma$ температура меняется монотонно, при $-0,8<\gamma<-0,2$ температура знакопеременна.

\section{4. Заключение}

В настоящей работе рассмотрены стационарные слоистые течения конвекции Бенара Марангони вязкой несжимаемой жидкости, индуцируемые градиентом температуры на обеих границах. Получены решения для краевых условий третьего рода на границах потока жидкости - теплообмен по закону Ньютона. Показано, что при некоторых условиях найдется 
толщина слоя жидкости, при которой касательные напряжения на твердой поверхности в направлении $x$ и $y$ одновременно обращаются в ноль.

\section{Благодарность}

Работа выполнена по теме государственного задания, ГР № 01201354600.

\section{Литература}

1. Thomson J. On certain curious motions observable on the surfaces of wine and other alcoholic liquours // Philosophical Magazine. - 1855. - T. 10. - P. 330-333.

2. Bénard H. Les Tourbillons cellulaires dans une nappe liquide propageant de la chaleur par convection, en régime permanent. - Paris : Gauthier-Villars, 1901.

3. B'enard H. Etude exp'erimentale des courants de convection dans une nappe liquide. R'egime permanent: tourbillons cellulaires // J. Phys. Theor. Appl. - 1900. - Vol. 9, no. 1. P. 513-524.

4. Гетлинг А. В. Формирование пространственных структур конвекции Рэлея-Бенара // Успехи физических наук. - 1991. - Т. 161, № 9. - С. 1-80.

5. Ланда П. С. Автоколебания в распределенных системах. - М. : Книжный дом "Либроком". - 2010. - $320 \mathrm{c}$.

6. Marangoni C. Sull espansione delle goccie di un liquido galleggiante sulla superficie di altro liquid // Pavia : Tipografia dei fratelli Fusi. - 1865.

7. Birikh R. V. Thermocappillary convection on a horizontal fluid layer // J. Appl. Mech. Technol. Phys. - 1966. - Vol. 7, iss. 3. - P. 43-44. - DOI: 10.1007/BF00914697.7.

8. Остроумов Г. А. Свободная конвекция в условиях внутренней задачи. - М.; Л. : Гостехтеоретиздат, 1952. - $256 \mathrm{c.}$

9. Гершуни Г. З., Жуховицкий Е. М. Конвективная устойчивость несжимаемой жидкости. - М. : Наука, 1972. - 392 с.

10. Napolitano L. G. Plane Marangoni-Poiseuille flow of two immissible fluids // Acta Astronautica. - 1980. - Vol. 7, iss. 4-5. - P. 461-478. - DOI: 10.1016/0094-5765(80)90036-3.

11. Сидоров А. Ф. Об одном классе решений уравнений газовой динамики и естественной конвекции. Численные и аналитические методы решения задач механики сплошной среды. : сборник научных трудов УНЦ АН СССР. - Свердловск : УНЦ АН СССР, 1981. C. 101-117.

12. Sidorov A. F. Two classes of solution of the fluid and gas mechanics equatins and their connection to traveling wave theory // Journal of Applied Mechanics and Technical Physics. - 1989. Vol. 30, iss. 2. - P. 197-203. - DOI: 10.1007/BF00852164.

13. Кирдяшкин А. Г. Тепловые гравитационные течения и теплообмен в астеносфере. Новосибирск : Наука, СО РАН, 1989. - 81 с.

14. Goncharova O., Kabov O. Gas flow and thermocapillary effects of fluid flow dynamics in a horizontal layer // Microgravity Sci. Technol. - 2009. - Vol. 21, iss. 1 suppl. - P. 129-137. DOI: $10.1007 / \mathrm{s} 12217-009-9108-\mathrm{x}$.

15. Андреев В. К. Решения Бириха уравнений конвекции и некоторые его обобщения. Препринт № 1-10. - Красноярск : ИВМ СО РАН, 2010. - 68 с.

16. Ingel L. Kh., Kalashnik Maksim V. Nontrivial features in the hydrodynamics of seawater and other stratified solutions // Physics-Uspekhi. - Vol. 55, no. 4. - P. 356-381. DOI: $10.3367 /$ UFNe.0182.201204b.0379.

17. Аристов С. Н., Шварц К. Г. Вихревые течения в тонких слоях жидкости. - Киров : ВятГУ, 2011. - 207 с.

18. Аристов С. Н., Просвиряков Е. Ю. О слоистых течениях плоской свободной конвекции // Нелинейная динамика. - 2013. - Т. 9, № 4. - С. 651-657. 
19. Шварц К. Г. Плоскопараллельное адвективное течение в горизонтальном слое несжимаемой жидкости с твердыми границами // Изв. РАН. МЖГ. - 2014. - № 4. - С.26-30.

20. Юдович В. И. О проблемах и перспективах современной математической гидродинамики // Успехи механики. - 2002. - Т. 1, № 1. - С. 61-102.

21. Аристов С. Н., Шварц К. Г. Вихревые течения адвективной природы во вращающемся слое жидкости. - Пермь : Изд-во ПГУ, 2006. - 154 с.

22. Aristov S. N., Shvarts K. G. Advective flow in a rotating liquid film // Journal of Applied Mechanics and Technical Physics. - 2016. - Vol. 57, no. 1. - P. 188-194. DOI: $10.1134 / \mathrm{S} 0021894416010211$.

23. Andreev V. K., Bekezhanova V. B. Stability of non-isothermal fluids (Review) // Journal of Applied Mechanics and Technical Physics. - 2013. - Vol. 54, iss. 2. - P. 171-184. DOI: $10.1134 / \mathrm{S} 0021894413020016$.

24. Пухначев В. В. Нестационарные аналоги решения Бириха // Известия Алтайского гос. ун-та. - 2011. - № 1-2. - С. 62-69.

25. Никитин Н. В., Никитин С. А., Полежаев В. И. Конвективные неустойчивости в гидродинамической модели роста кристаллов методом Чохральского // Успехи механики. 2003. - Т. 2, № 4. - С. 63-105.

26. Аристов С. Н., Зимин В. Д. Адвективные волны во вращающемся шаровом слое: Препринт № 145. - Свердловск : ИМСС, Уральский научный центр, АН СССР, 1986. - 50 с.

27. Аристов С. Н., Фрик П. Г. Динамика крупномасштабных течений в тонких слоях жидкости: Препринт № 146. - Свердловск : ИМСС, Уральский научный центр, АН СССР, 1987. $48 \mathrm{c}$.

28. Аристов С. Н., Шварц К. Г. Конвективный теплообмен при локализованном нагреве плоского слоя несжимаемой жидкости // Изв. РАН. МЖГ. - 2013. - № 3. - С. 53-58.

29. Аристов С. Н., Просвиряков Е. Ю. Об одном классе аналитических решений стационарной осесимметричной конвекции Бенара-Марангони вязкой несжимаемой жидкости // Вестн. Сам. гос. техн. ун-та. Сер. физ.-мат. науки. - 2013. - № 3 (32). - С. 110-118.

30. Аристов С. Н., Князев Д. В. Локализованные конвективные течения в слое неоднородно нагретой жидкости // Изв. РАН. МЖГ. - 2014. - № 5. - С. 5-16.

31. Аристов С. Н., Фрик П. Г. Крупномасштабная турбулентность в тонком слое неизотермической вращающейся жидкости // Изв. АН СССР. МЖГ. - 1988. - № 4. - С. 48-55.

32. Ландау Л. Д. Теоретическая физика. Т. 6. Гидродинамика. - М. : Наука, 2006. - 736 с.

33. Lin C. C. Note on a class of exact solutions in magneto-hydrodynamics // Arch. Rational Mech. Anal. - 1958. - Vol. 1. - P. 391-395.

34. Аристов С. Н., Князев Д. В., Полянин А. Д. Точные решения уравнений Навье-Стокса с линейной зависимостью компонент скорости от двух пространственных переменных // Теорет. основы хим. технологии. - 2009. - Т. 43, № 5. - С. 547-566.

35. $\quad$ Гантмахер Ф. Р. Теория матриц. - М. : Наука, 1967. - 576 с. 